MS

Editions

Sociedad Latinoamericana de Plantas Medicinales www.mpc.ms-editions.cl

MEDICINAL PLANT COMMUNICATIONS

Med Plant Commun

3 (4): 74 - 78 (2020) - https://doi.org/10.37360/mpc.20.3.4.14

(C) / ISSN 24524433

\title{
HPLC-ESI-QTOF-MS/MS based phytochemical characterization: estrogenic and antioxidant effect of Mangifera indica L. cv. Kent on vaginal and bone tissue of ovariectomized rats
}

[Caracterización fitoquímica basada en HPLC-ESI-QTOF-MS/MS: efecto estrogénico y antioxidante de Mangifera indica L. cv. Kent sobre tejido óseo y vaginal de ratas ovariectomizadas]

Roberto O. Ybañez-Julca1, Daniel Asunción-Alvarez ${ }^{1}$, Julio Benites², Ricardo D.D.G. de Albuquerque ${ }^{3}$, Leandro Rocha ${ }^{3} \&$ José L. Martinez ${ }^{4}$

${ }^{1}$ Laboratorio de Farmacología, Facultad de Farmacia y Bioquímica, Universidad Nacional de Trujillo, Trujillo, Perú

${ }^{2}$ Química y Farmacia, Facultad de Ciencias de la Salud, Universidad Arturo Prat, Iquique, Chile

${ }^{3}$ Laboratório de Tecnologia em productos Naturais, Universidade Federal Fluminense, Niterói, Brasil

${ }^{4}$ Vicerrectoria de Investigación, Desarrollo e Innovación, Universidad de Santiago de Chile, Chile

rybanez@unitru.edu.pe

\begin{abstract}
The phytochemical characterization of the hydroalcoholic extract of Mangifera indica leaves L. cv. Kent (MS2) was determined by HPLC-ESI-QTOF-MS/MS. Eight metabolites were identified, these include: benzophenone derivatives and xanthones. Using ovariectomized rats $(\mathrm{OVX})$, our findings suggest that EM2 exhibited an estrogenic effect by increasing the number of cornified cells in vaginal tissue. Furthermore, EM2 demonstrated a high antioxidant effect by reducing malondialdehyde (MDA) levels in OVX rat bone. We conclude that the polyphenolic compounds present in EM2 could increase the number of cornified cells and act as exogenous antioxidant agents against oxidative damage in OVX rats.
\end{abstract}

Keywords: Mangifera indica L; Benzophenone derivatives; Lipid peroxidation; Ovariectomy; Antioxidants

Resumen: La caracterización fitoquímica del extracto hidroalcohólico de hojas de Mangifera indica L. cv. Kent (EM2) se determinó mediante HPLC-ESI-QTOF-MS/MS. Se identificaron ocho metabolitos, estos incluyen: derivados de benzofenona y xantonas. Usando ratas ovariectomizadas (OVX), nuestros hallazgos sugieren que EM2 exhibió un efecto estrogénico al aumentar el número de células cornificadas en el tejido vaginal. Además, EM2 demostró un alto efecto antioxidante al reducir los niveles de malondialdehído (MDA) en el hueso de rata OVX. Concluimos que los compuestos polifenólicos presentes en EM2 podrían aumentar el número de células cornificadas y actuar como agentes antioxidantes exógenos contra el daño oxidativo en ratas OVX.

Palabras clave: Mangifera indica L; Derivados de benzofenona; Peroxidación lipídica; Ovariectomía; Antioxidantes.

Received: 23 de marzo de 2020

Accepted: 15 de mayo de 2020

Published online: 30 de noviembre de 2020

This article must be cited as: Ybañez-Julca RO, Asunción-Alvarez D, Benites J, de Albuquerque RDDG, Rocha L, Martinez JL. 2020. HPLC-ESI-QTOF-MS/MS based phytochemical characterization: estrogenic and antioxidant effect of Mangifera indica L. cv. Kent on vaginal and bone tissue of ovariectomized rats. Med Plant Commun 3 (4): 74 $-78$ 


\section{INTRODUCCIÓN}

Las mujeres viven más que los hombres, debido a que los estrógenos regulan positivamente los genes antioxidantes relacionados con la longevidad [1]. Sin embargo, los cambios hormonales que comienzan durante la transición menopáusica afectan muchos sistemas biológicos [2]. Esto trae consigo la aparición de síntomas físicos como debilidad, sofocos, sudoración nocturna, atrofia urogenital, disfunción sexual, cambios en el estado de ánimo, pérdida ósea y cambios metabólicos [3,4]. Además, los bajos niveles de estrógeno pueden ocasionar un desequilibrio redox, condición en la que las especies reactivas de oxígeno (ROS) y los prooxidantes superan la capacidad antioxidante, generando un daño celular grave [5]. Como resultado de esto, se produce una amplia variedad de productos tóxicos como el malondialdehido (MDA) [6].

Aunque la terapia de reemplazo hormonal (TRH) sigue siendo el tratamiento más efectivo para aliviar los síntomas de la menopausia [7], se han reportado numerosos efectos adversos, así como contraindicaciones y riesgos, esto ha incentivado la búsqueda nuevas alternativas terapéuticas que tengan una mejor seguridad y tolerancia [8]. La búsqueda de moléculas bioactivas extraídas de plantas medicinales utilizadas por etnias indígenas está ganando mucha consideración como fuente de nuevas moléculas para el tratamiento de diversas enfermedades. Mangifera indica L. "mango" (familia Anacardiaceae) tiene un gran potencial farmacológico, ya que, según se informa, se destaca como anticanceroso, antiinflamatorio, antidiabético, antioxidante, antibacteriano, gastroprotector, hepatoprotector, antihiperlipémico, antiespasmódico, entre otros [9,10]. Sin embargo, a pesar de que se tiene importante evidencia sobre sus propiedades antioxidantes y contenido de fitoestrógenos in vitro; son muy pocos los estudios que corroboren y relacionen este efecto en modelos experimentales in vivo [10,11,12]. Por ello, la presente investigación tuvo por objetivo determinar el efecto de la administración de Mangifera indica L. cv. Kent sobre parámetros estrogénicos como el número de células cornificadas y sobre la lipoperoxidación en tejido óseo de ratas ovariectomizadas.

\section{METODOLOGÍA}

\section{Preparación del extracto}

Se preparó un extracto con etanol 50\% y se llevó a un equipo de reflujo por dos horas. Los extractos se filtraron y luego se evaporaron a presión reducida. El extracto se congeló a $-80^{\circ} \mathrm{C}$ y luego se liofilizó. El extracto hidroalcohólico liofilizado de hojas de Mangifera indica L. cv. Kent (EM2) se almacenó a $4^{\circ} \mathrm{C}$ hasta que se analizó.

\section{Caracterización fitoquímica por HPLC-ESI-QTOF-MS/MS}

La identificación química se realizó utilizando un espectrómetro de masas ortogonal Q-TOF (micrOTOF-QTM, Bruker Daltonics) equipado con una fuente de ionización por electroaspersión (ESI). Los parámetros de análisis se proporcionaron para el modo positivo, con un rango de masa de 100-1000 m / z: voltaje capilar de $4500 \mathrm{~V}$; ajuste la placa final offset -500 V; ajuste el voltaje de carga $2000 \mathrm{~V}$; temperatura del gas de secado $200^{\circ} \mathrm{C}$; flujo de gas de secado 10,0 ml / min; presión de gas 4 bar; energía de colisión (MS/MS) 35 eV; colisión de gas N2 [13]. Los datos de masa obtenidos se procesaron en el software Bruker Compass Data Analysis 4.2 (Bruker Daltonics).

\section{Ovariectomía: Técnica de Laparotomía medía}

Los especímenes fueron anestesiados para poder extirpar los ovarios. Una vez extirpados los cuernos uterinos se colocaron en su posición inicial y se procedió al cierre de la cavidad abdominal. La herida se observó diariamente para confirmar la ausencia de complicaciones hasta su cicatrización [13].

\section{Tratamientos animales}

Los animales de experimentación fueron divididos en 4 grupos: Naive $(\mathrm{n}=6)$, conformado por ratas no ovariectomizadas (no OVX); Control $(\mathrm{n}=6$ ), ratas OVX tratadas vía oral $(\mathrm{VO})$ con $\mathrm{NaCl}$ 0,9\%; y los grupos Problema $\mathrm{I}(\mathrm{n}=6)$ y Problema II $(\mathrm{n}=6)$ que fueron ratas OVX a las cuales se les administró VO el liofilizado (EM2) en dosis de $250 \mathrm{mg} / \mathrm{kg}$ y $500 \mathrm{mg} / \mathrm{kg}$ respectivamente, durante 28 días [13].

\section{Citología vaginal}

Las células se recogieron del canal vaginal con la ayuda de un hisopo. La muestra se fijó con etanol durante 15 minutos y 
luego se almacenó a $27^{\circ} \mathrm{C}$. La tinción se realizó con hematoxilina-eosina (H\&E). Luego, las muestras fueron analizadas microscópicamente [13].

\section{Niveles de peroxidación lipídica en tejido óseo}

El tejido óseo (fémur) se removió y después de ser limpiados, se trituraron y mezclaron vigorosamente con una solución fría de cloruro de potasio $\left(4^{\circ} \mathrm{C}\right)(\mathrm{KCl}, 150 \mathrm{mM})$ para obtener un homogeneizado. Los homogeneizados se centrifugaron a $3700 \mathrm{~g}\left(4^{\circ} \mathrm{C}\right)$ durante $15 \mathrm{~min}$. Los sobrenadantes se tomaron para el análisis de malondialdehido de acuerdo al método de las sustancias reactivas al ácido tiobarbitúrico (TBARS) a $532 \mathrm{~nm}$ [14].

\section{RESULTADOS Y DISCUSIÓN}

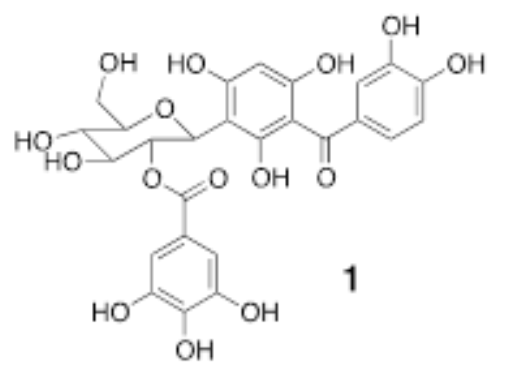

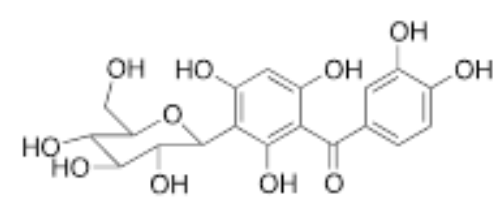

2

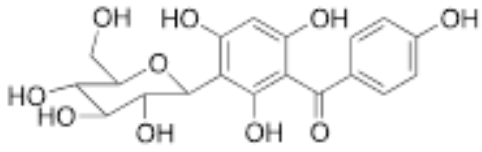

3

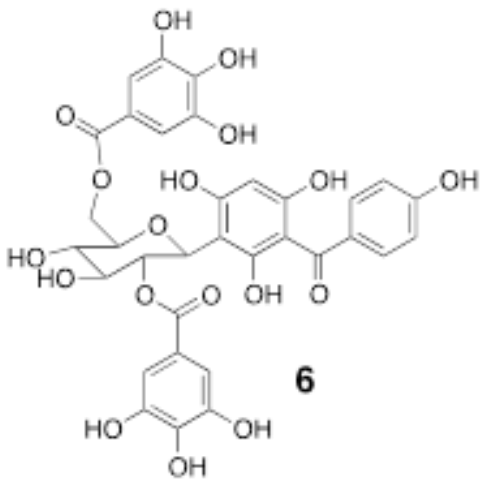<smiles>COc1c(C(=O)c2ccc(O)cc2)c(O)cc(O)c1C(COCC(O)COC(=O)c1cc(O)c(O)c(O)c1)OC(=O)c1cc(O)c(O)c(O)c1</smiles><smiles>O=C(OCCOC(O)C(O)OCCOCCO)c1cc(O)c(O)c2c(=O)c3cc(O)c(O)cc3oc12</smiles>

8

Figura $\mathbf{N}^{\mathbf{0}} 1$

Estructuras de los compuestos fenólicos presentes en las hojas de Mangifera indica L. cv. Kent.

Las estructuras de los metabolitos secundarios identificados en las hojas de mango (Mangifera indica L. cv. Kent) se muestran en la Figura $\mathrm{N}^{\circ}$ 1. El análisis HPLC-ESI-QTOF-MS/MS a partir de extractos de metanol (MeOH) de hoja de mango mostró compuestos polifenólicos caracterizados como derivados de benzofenona y xantonas.

Se identificaron seis derivados de benzofenona a partir del extracto de $\mathrm{MeOH}$ (1, isómero de la maclurina 3-C-(2-Ogaloyil)-D-glucósido; 2 , maclurina 3-C- $\beta$-D-glucósido; 3, iriflofenona 3-C- $\beta$-D-glucósido; 5 , maclurina 3-C-(2,3-di-Ogalloíl)- $\beta$-D-glucósido; 6, iriflofenona 3-C-(2-O-galloil)- $\beta$-D-glucósido; 7, metil-iriflofenona 3-C-(2,6-di-O-galloyl)- $\beta$-Dglucósido) como se muestra en la Figura $\mathrm{N}^{\circ}$. Asimismo, se detectaron dos derivados de xantonas en el extracto 
$\mathrm{MeOH}$ de las hojas de Mangifera indica L cv. Kent. Los picos en m/z 423 y 575 correspondieron a iones $[\mathrm{M}+\mathrm{H}]+$ y se atribuyeron a mangiferina (4) y 6-O-galloil-mangiferina (8) respectivamente.
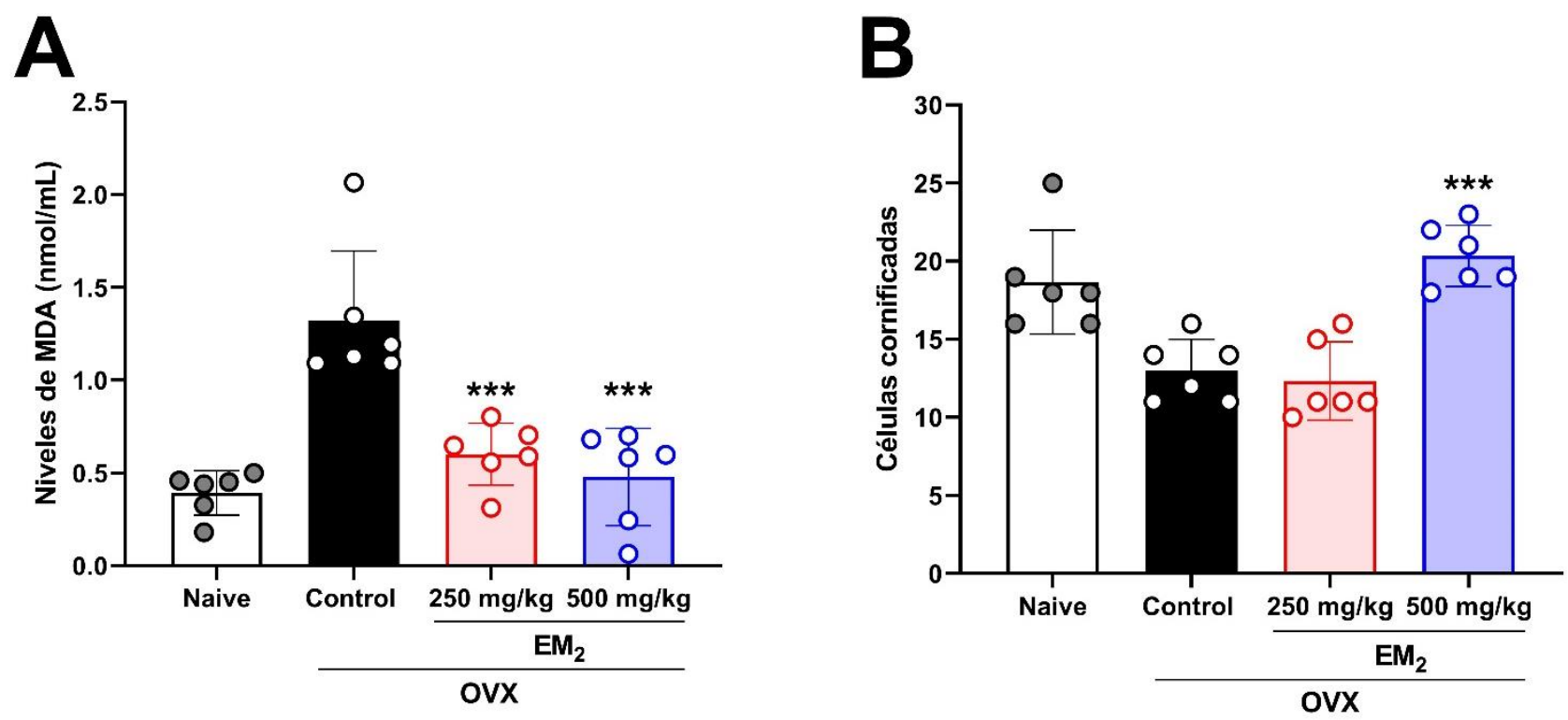

Figura $\mathbf{N}^{\mathrm{o}} 2$

Efecto del tratamiento oral con el liofilizado de hojas de Mangifera indica L. cv. Kent (EM2) durante 28 días sobre los niveles de MDA en tejido óseo (A) y el conteo de células cornificadas en tejido vaginal (B). $* * * p<0,001 * * * * p<0,0001$ vs OVX (control).

Los resultados muestran que la administración de EM2 aumenta significativamente ( $500 \mathrm{mg} / \mathrm{kg}$; $p<0,001)$ el número de células cornificadas en ratas OVX. Reportes previos ha sugerido que la mangiferina, uno de los componentes identificados en el extracto de Mangifera indica, es capaz de activar una de las isoformas del receptor de estrógeno $(\mathrm{ER} \alpha)$ misma que está implicada en procesos de proliferación celular [14]. Además, existe evidencia de que ER $\alpha$ es un receptor estrogénico clave para la regulación del receptor de oxitocina OTR en útero de rata, además que también estaría implicado en la regulación de OTR durante el parto [15].

Finalmente, EM2 exhibió un potente efecto antioxidante in-vivo, ya que redujo significativamente ( $250 \mathrm{mg} / \mathrm{kg}$; $p<0,001 \mathrm{y}$ $500 \mathrm{mg} / \mathrm{kg} ; p<0,001)$ los niveles de MDA en tejido óseo de ratas OVX. La presencia de mangiferina tal como lo reporta el trabajo realizado por Xia et al. estaría confiriendo un efecto protector contra el daño oxidativo sobre los osteoblastos mediante la modulación de la señalización ERK5/Nrf2[16].

\section{CONCLUSIÓN}

El extracto liofilizado de hojas de Mangifera indica L. cv. "Kent" exhibe efectos estrogénicos y antioxidante en tejido vaginal y óseo respectivamente, posiblemente debido a la presencia de compuestos polifenólicos como derivados de benzofenonas y xantonas.

\section{AGRADECIMIENTOS}

Agradecemos al Fondo Nacional de Desarrollo Científico, Tecnológico y de Innovación Tecnológica (FONDECYT) del Consejo Nacional de Ciencia Tecnología e Innovación Tecnológica (CONCYTEC-Perú) Becas N N 153-2015FONDECYT y a la subunidad RPT13B - Métodos analíticos del Instituto de Tecnología de Drogas (FIOCRUZ, Brasil), por el apoyo financiero a este estudio. 


\section{REFERENCIAS}

[1] Viña J et al. 2008. Biol Chem 389: 273-277. https://doi.org/10.1515/bc.2008.027

[2] Monteleone P et al. 2018. Nature Rev Endocrinol 14: 199-215. https://doi.org/10.1038/nrendo.2017.180

[3] Auro K et al. 2014. Nature Commun 5. https://doi.org/10.1038/ncomms5708

[4] Davis SR et al. 2015. Nature Rev Dis Prim 1: 1-19. https://doi.org/10.1038/nrdp.2015.4

[5] Can S et al. 2015. Hepat Mon 15: e22633. https://doi.org/10.5812/hepatmon.22633

[6] Ayala A et al. 2014. Oxid Med Cell Longev 2014: 1-31. https://doi.org/10.1155/2014/360438

[7] Hickey $\mathrm{M}$ et al. 2005. The Lancet 366: 409-421. https://doi.org/10.1016/s0140-6736(05)66519-1

[8] Hickey M et al. 2012. BMJ 344: e763. https://doi.org/10.1136/bmj.e763

[9] Ediriweera MK et al. 2017. Evid Based Compl Alternat Med 2017: 6949835 https://dpi.org/10.1155/2017/6949835

[10] Ybañez-Julca RO et al. 2020. Molecules 25: 5149. https://doi.org/10.3390/molecules25215149

[11] Asunción-Álvarez D et al. 2018. Rev Peru Med Integrat 3: 24-31. https://doi.org/10.26722/rpmi.2018.31.77

[12] Ribeiro S et al. 2010. Bioact Foods Promot Health 507-523. https://doi.org/0.1016/b978-0-12-374628-3.00034-7

[13] Benites J et al. 2019. Bol Latinoam Caribe Plantas Med Aromat 18: 336-349.

[14] Wilkinson AS et al. 2015. Food Funct 6: 1847-1854. https://doi.org/10.1039/c5fo00133a

[15] Murata T et al. 2013. J Reprod Dev 60: 55-61. https://doi.org/10.1262/jrd.2012-139

[16] Xia G et al. 2017. Biochem Biophys Res Commun 491: 807-813. https://doi.org/10.1016/j.bbrc.2017.06.184 